\title{
Pulsed electromagnetic field radiation from a narrow slot antenna with a dielectric layer
}

\author{
M. Štumpf, ${ }^{1}$ A. T. de Hoop, ${ }^{2}$ and I. E. Lager ${ }^{3}$ \\ Received 27 November 2009; revised 29 April 2010; accepted 27 May 2010; published 5 October 2010.
}

[1] Analytic time domain expressions are derived for the pulsed electromagnetic field radiated by a narrow slot antenna with a dielectric layer in a two-dimensional model configuration. In any finite time window of observation, exact pulse shapes for the propagated, reflected, and refracted wave constituents are constructed with the aid of the modified Cagniard method (Cagniard-DeHoop method). Numerical results are presented for vanishing slot width and field pulse shapes at the dielectric/free space interface. Applications are found in any system whose operation is based on pulsed electromagnetic field transfer and where digital signals are detected and interpreted in dependence on their pulse shapes.

Citation: Štumpf, M., A. T. de Hoop, and I. E. Lager (2010), Pulsed electromagnetic field radiation from a narrow slot antenna with a dielectric layer, Radio Sci., 45, RS5005, doi:10.1029/2009RS004335.

\section{Introduction}

[2] With the rapid development of communication systems whose operation is based upon the transfer of pulsed electromagnetic fields and the detection and subsequent interpretation of the pertaining digital signals, there is a need for mathematical analysis of model configurations where the influence of (a number of) the system parameters on the performance shows analytic expressions in analytic time domain that characterize the physical behavior. The present paper aims at providing such a tool with regard to the pulsed radiation behavior of a narrow slot antenna covered with a dielectric layer in a two-dimensional setting. The source exciting the structure is modeled as a prescribed distribution of the transverse electric field across a narrow slot of uniform width in a perfectly electrically conducting planar screen. The pulse shape of the exciting field is arbitrary. In front of this slotted plane, there is a homogeneous, isotropic

\footnotetext{
${ }^{1}$ Department of Radio Electronics, Faculty of Electrical Engineering and Communication, Brno University of Technology, Brno, Czech Republic.

${ }^{2}$ Laboratory of Electromagnetic Research, Faculty of Electrical Engineering, Mathematics and Computer Science, Delft University of Technology, Delft, Netherlands.

${ }^{3}$ International Research Centre for Telecommunications and Radar, Faculty of Electrical Engineering, Mathematics and Computer Science, Delft University of Technology, Delft, Netherlands.

Copyright 2010 by the American Geophysical Union. 0048-6604/10/2009RS004335
}

dielectric slab of uniform thickness. The structure further radiates into free space. Using the combination of a unilateral Laplace transformation with respect to time and the spatial slowness representation of the field components that is known as the modified Cagniard method (CagniardDeHoop method), analytic time domain expressions are obtained for the electric and the magnetic field as a function of position and time. The representation appears as the superposition of a number of propagating, reflecting, and refracting wave constituents in the slab and is, within any finite time window of observation, exact. It is immediately clear that the pulse shapes of these constituents (that successively reach a receiving observer) are distorted versions of the activating source signature. Parameters in this respect are the pulse shape of the excitation (characterized by the pulse risetime and the pulse time width of a unipolar pulse), the thickness and the dielectric properties of the slab, as well as the position of observation relative to the exciting slot.

[3] The analytic expressions are readily evaluated numerically. Results are presented for vanishing slot width and field pulse shapes at the dielectric/free space interface for a variety of parameters, all chosen such that the pulse time width is smaller than the travel time needed to traverse the slab. In this way, the study can focus on the changes in pulse shape that occur in the individual successive wave constituents. In line with the International Electrotechnical Vocabulary (IEV) of the International Electrotechnical Committee (IEC 60050-IEV) (http://www.electropedia.org), the signature of the excitation is taken to be a unipolar pulse charac- 


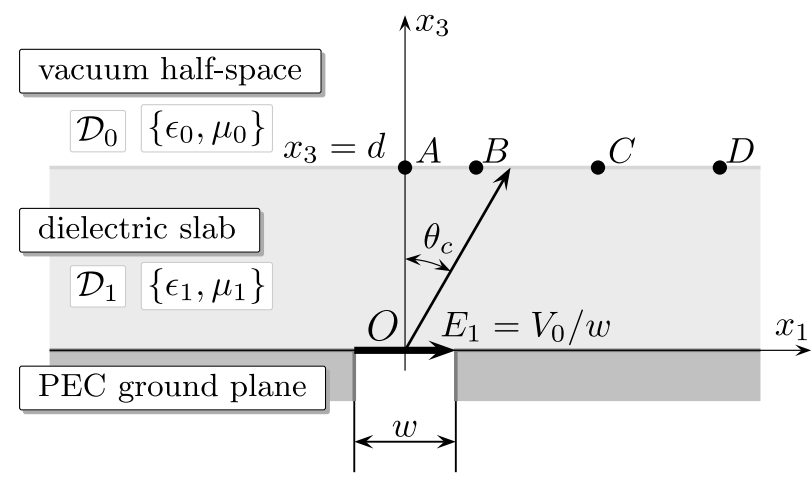

Figure 1. Configuration with indication of the critical angle $\theta_{c}=\arcsin \left(c_{1} / c_{0}\right)$. Positions of observation points $\{\mathrm{B}, \mathrm{C}, \mathrm{D}\}$ along the line $\left\{x_{3}=d\right\}$ are not true-to-scale with those chosen in section 6 .

terized by its pulse amplitude, pulse risetime, and pulse time width. The power exponential pulse provides a convenient mathematical model to accommodate these parameters.

[4] Apart from this, the obtained expressions can serve the purpose of benchmarking the performance of purely computational techniques that have to be called upon in the more complicated configurations met in practice, in particular the ones in patch antenna design, where the field calculated in the present paper represents the field "incident" on the geometry of the patches.

\section{Description of the Configuration and Formulation of the Field Problem}

[5] The configuration examined is shown in Figure 1. In Figure 1, the position is specified by the right-handed orthogonal Cartesian coordinates $\left\{x_{1}, x_{2}, x_{3}\right\}$. The time coordinate is $t$. Partial differentiation with respect to $x_{m}$ is denoted by $\partial_{m} ; \partial_{t}$ is a reserved symbol denoting partial differentiation with respect to $t$. The configuration consists of an unbounded electrically perfectly conducting screen $\mathcal{S}=\left\{\left(-\infty<x_{1}<-w / 2\right) \bigcup\left(w / 2<x_{1}<\infty\right),-\infty<x_{2}\right.$ $\left.<\infty, x_{3}=0\right\}$ with a feeding aperture $\mathcal{A}=\left\{-w / 2<x_{1}<w /\right.$ $\left.2,-\infty<x_{2}<\infty, x_{3}=0\right\}$ of width $w \downarrow 0$. The covering dielectric slab occupies the domain $\mathcal{D}_{1}=\left\{-\infty<x_{1}<\infty\right.$, $\left.-\infty<x_{2}<\infty, 0<x_{3}<d\right\}$. The structure radiates into the vacuum half-space $\mathcal{D}_{0}=\left\{-\infty<x_{1}<\infty,-\infty<x_{2}<\infty, d<\right.$ $\left.x_{3}<\infty\right\}$. The spatial distribution of electric permittivity and magnetic permeability is

$$
\{\epsilon, \mu\}= \begin{cases}\left\{\epsilon_{0}, \mu_{0}\right\} & \text { in } \mathcal{D}_{0} \\ \left\{\epsilon_{1}, \mu_{1}\right\} & \text { in } \mathcal{D}_{1} .\end{cases}
$$

The corresponding electromagnetic wave speeds are $c_{0}=$ $\left(\epsilon_{0} \mu_{0}\right)^{-1 / 2}$ and $c_{1}=\left(\epsilon_{1} \mu_{1}\right)^{-1 / 2}$. The antenna aperture is fed by the uniformly distributed, $x_{2}$-independent, electric field

$$
E_{1}\left(x_{1}, 0, t\right)=V_{0}(t) / w \text { in } \mathcal{A},
$$

where $V_{0}(t)$ is the feeding "voltage." Since the excitation, as well as the configuration, are independent of $x_{2}$, the nonzero components of the electric field strength $\left\{E_{1}, E_{3}\right\}$ $\left(x_{1}, x_{3}, t\right)$ and the magnetic field strength $H_{2}\left(x_{1}, x_{3}, t\right)$ satisfy in $\mathcal{D}_{0}$ and $\mathcal{D}_{1}$ the source-free field equations:

$$
\partial_{1} H_{2}-\epsilon \partial_{t} E_{3}=0
$$

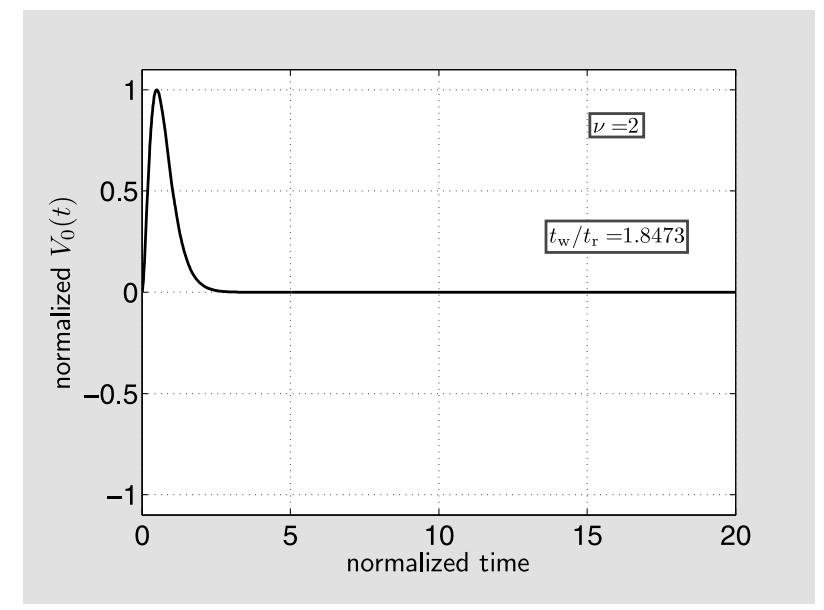

a

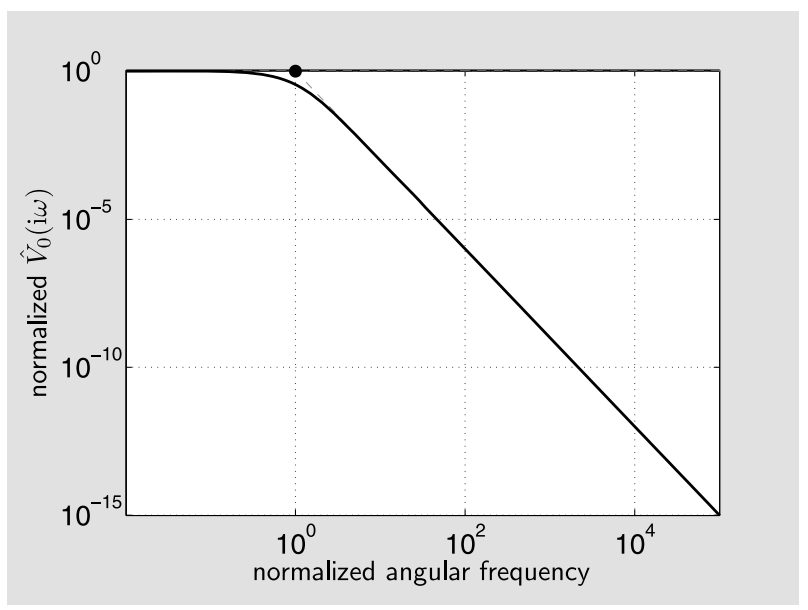

$\mathrm{b}$

Figure 2. The power exponential excitation signature: (a) pulse shape and (b) spectral diagram. The represented quantities are $V_{0}(t) / V_{\max }$ (normalized $\left.V_{0}(t)\right), c_{0} t / d$ (normalized time), $\hat{V}_{0}(1 \omega) / \hat{V}_{0}(0)$, (normalized $\hat{V}_{0}(1 \omega)$ ), $\omega / \omega_{\text {corner }}$ (normalized angular frequency). 
Table 1. Arrival Times of Time Domain Constituents

\begin{tabular}{ccccccccc}
\hline & \multicolumn{3}{c}{$c_{0} T_{\mathrm{HW}}^{n]} / d$} & \multicolumn{5}{c}{$c_{0} T_{\mathrm{BW}}^{n]} / d$} \\
\cline { 2 - 8 } Order $n$ & $\mathrm{~A}$ & $\mathrm{~B}$ & $\mathrm{C}$ & $\mathrm{D}$ & $\mathrm{A}$ & $\mathrm{B}$ & $\mathrm{C}$ & $\mathrm{D}$ \\
\hline 0 & - & - & 4.5321 & 6.7231 & 2.0 & 2.2361 & 5.9464 & 10.1980 \\
1 & - & - & 7.9962 & 10.1962 & 6.0 & 6.0828 & 8.2073 & 11.6619 \\
2 & - & - & - & 13.6603 & 10.0 & 10.0499 & 11.4612 & 14.1421 \\
3 & - & - & - & 17.1244 & 14.0 & 14.0357 & 15.0785 & 17.2047 \\
4 & - & - & - & $>20$ & 18.0 & 18.0278 & 18.8510 & $>20$ \\
\hline
\end{tabular}

$$
\begin{gathered}
\partial_{3} H_{2}+\epsilon \partial_{t} E_{1}=0, \\
\partial_{1} E_{3}-\partial_{3} E_{1}-\mu \partial_{t} H_{2}=0 .
\end{gathered}
$$

The interface boundary conditions require that

$$
\begin{aligned}
& \lim _{x_{3} \downarrow d} E_{1}=\lim _{x_{3} \uparrow d} E_{1} \quad \text { for all } x_{1} \text { and } t \\
& \lim _{x_{3} \downarrow d} H_{2}=\lim _{x_{3} \uparrow d} H_{2} \quad \text { for all } x_{1} \text { and } t,
\end{aligned}
$$

while the excitation condition is

$$
\begin{gathered}
\lim _{x_{3} \downarrow 0} E_{1}=\left[V_{0}(t) / w\right] \\
{\left[H\left(x_{1}+w / 2\right)-H\left(x_{1}-w / 2\right)\right] \text { for all } t}
\end{gathered}
$$

with $H(x)$ denoting the Heaviside unit step function. It is assumed that $V_{0}(t)$ starts to act at $t=0$ and that prior to this instant, the field vanishes throughout the configuration.

\section{Field Representations}

[6] Analytic time domain expressions for the field components will be constructed with the senior (second) author's modification of the Cagniard method (CagniardDeHoop method) [Cagniard, 1939; Cagniard, 1962; De Hoop, 1960; De Hoop and Frankena, 1960; Langenberg, 1974; De Hoop, 1979; De Hoop and Oristaglio, 1988; De Hoop, 2000]. The method employs a unilateral Laplace transformation with respect to time of the type

$$
\hat{V}_{0}(s)=\int_{t=0}^{\infty} \exp (-s t) V_{0}(t) \mathrm{d} t
$$

in which $s$ is taken to be real-valued and positive. In fact, Lerch's theorem [Widder, 1946] states that uniqueness of the inverse transformation is ensured under the weaker condition that $\hat{V}_{0}(s)$ is specified at the sequence of real $s$ values $\left\{s \in \mathbb{R} ; s_{n}=s_{0}+n h, s_{0}>0, h>0, n=0,1,2, \ldots\right\}$. The next step is to use the slowness representation of the field quantities

$$
\begin{aligned}
\left\{\hat{E}_{1}, \hat{H}_{2}, \hat{E}_{3}\right\}\left(x_{1}, x_{3}, s\right) \\
=\frac{s}{2 \pi \mathrm{i}} \int_{p=-\mathrm{i} \infty}^{\mathrm{i} \infty} \exp \left(-s p x_{1}\right) \\
\quad \cdot\left\{\tilde{E}_{1}, \tilde{H}_{2}, \tilde{E}_{3}\right\}\left(p, x_{3}, s\right) \mathrm{d} p
\end{aligned}
$$

that involves imaginary values of the complex slowness parameter $p$. Using (9) and (10), the field equations (3)-(5) transform into

$$
-s p \tilde{H}_{2}-s \epsilon \tilde{E}_{3}=0
$$

$$
\partial_{3} \tilde{H}_{2}+s \epsilon \tilde{E}_{1}=0
$$

$$
-s p \tilde{E}_{3}-\partial_{3} \tilde{E}_{1}-s \mu \tilde{H}_{2}=0,
$$

the interface boundary conditions (6) and (7) into

$$
\begin{aligned}
& \lim _{x_{3} \downarrow d} \tilde{E}_{1}=\lim _{x_{3} \uparrow d} \tilde{E}_{1} \\
& \lim _{x_{3} \downarrow d} \tilde{H}_{2}=\lim _{x_{3} \uparrow d} \tilde{H}_{2},
\end{aligned}
$$

and the excitation condition (8) into

$$
\lim _{x_{3} \downarrow 0} \tilde{E}_{1}=\frac{\hat{V}_{0}(s)}{w} \frac{\exp (s p w / 2)-\exp (-s p w / 2)}{s p} .
$$

In what follows we shall consider the limiting case of vanishing slot width. Then, (16) reduces to

$$
\lim _{x_{3} \downarrow 0} \tilde{E}_{1}=\hat{V}_{0}(s) \text {. }
$$

The slowness-domain field quantities follow from (11) (17) by expressing them in the form

$$
\begin{aligned}
\left\{\tilde{E}_{1}, \tilde{H}_{2}, \tilde{E}_{3}\right\}\left(p, x_{3}, s\right) & \\
=\{ & \left.\gamma_{0}(p) / \epsilon_{0}, 1,-p / \epsilon_{0}\right\} A_{0}^{+}(p, s) \\
& \cdot \exp \left[-s \gamma_{0}(p)\left(x_{3}-d\right)\right] \text { in } \mathcal{D}_{0}
\end{aligned}
$$




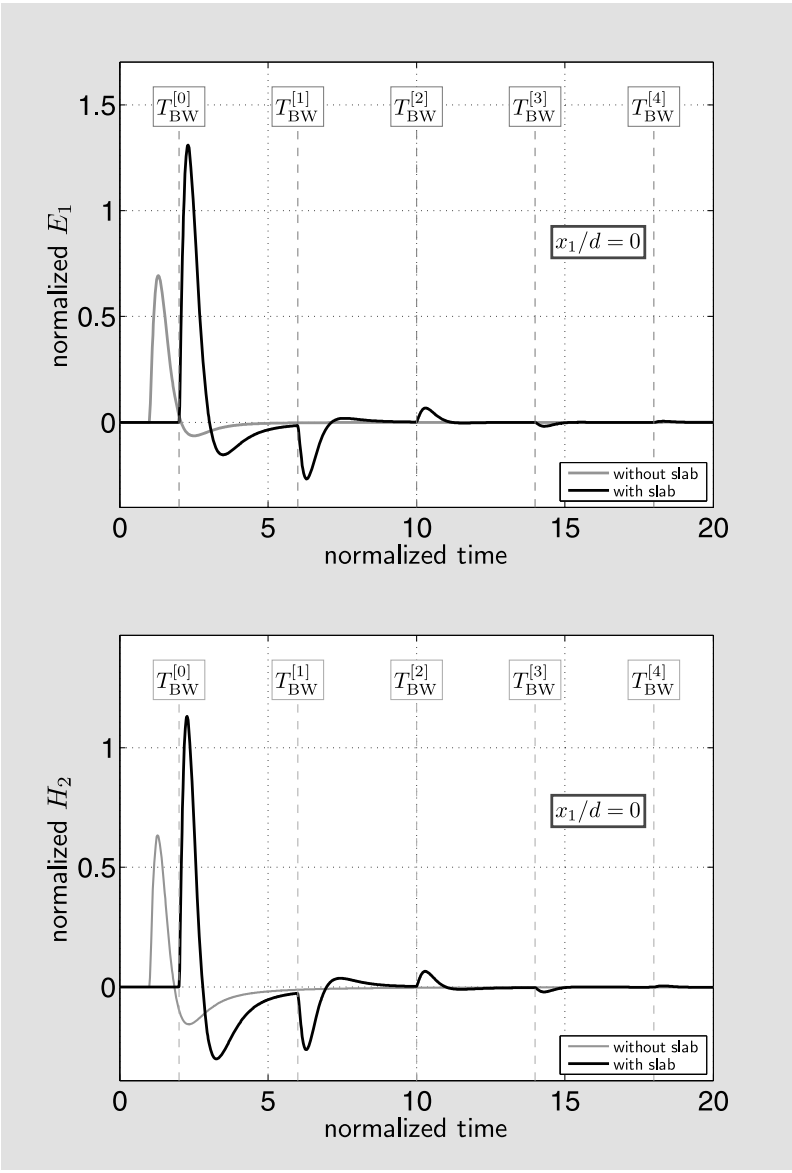

Figure 3. Time domain responses at $x_{1} / d=0$. The represented quantities are $E_{1} d / V_{\max }$ normalized $\left.E_{1}\right),\left(\mu_{0} / \epsilon_{0}\right)^{1 / 2}$ $\mathrm{H}_{2} d / V_{\max }$ (normalized $\mathrm{H}_{2}$ ), and $c_{0} t / d$ (normalized time).

$$
\begin{aligned}
\left\{\tilde{E}_{1}, \tilde{H}_{2}, \tilde{E}_{3}\right\}\left(p, x_{3}, s\right) & \\
= & \left\{\gamma_{1}(p) / \epsilon_{1}, 1,-p / \epsilon_{1}\right\} A_{1}^{+}(p, s) \exp \left[-s \gamma_{1}(p) x_{3}\right] \\
& +\left\{-\gamma_{1}(p) / \epsilon_{1}, 1,-p / \epsilon_{1}\right\} A_{1}^{-}(p, s) \\
& \cdot \exp \left[-s \gamma_{1}(p)\left(d-x_{3}\right)\right] \quad \text { in } \mathcal{D}_{1}
\end{aligned}
$$

in which

$$
\gamma_{0,1}(p)=\left(1 / c_{0,1}^{2}-p^{2}\right)^{1 / 2}
$$

with $\operatorname{Re}\left[\gamma_{0,1}(p)\right]>0$ for all $p \in \mathbb{C}$. Using these expressions in (14), (15), and (17) it is found that

$$
A_{1}^{+}(p, s)=\frac{\epsilon_{1}}{\gamma_{1}(p)} \frac{\hat{V}_{0}(s)}{\Delta}
$$

$$
\begin{aligned}
& A_{1}^{-}(p, s)=\frac{\epsilon_{1}}{\gamma_{1}(p)} R_{H}(p) \frac{\hat{V}_{0}(s) \exp \left[-s \gamma_{1}(p) d\right]}{\Delta} \\
& A_{0}^{+}(p, s)=\frac{\epsilon_{1}}{\gamma_{1}(p)} T_{H}(p) \frac{\hat{V}_{0}(s) \exp \left[-s \gamma_{1}(p) d\right]}{\Delta}
\end{aligned}
$$

in which

$$
R_{H}(p)=\frac{\gamma_{1}(p) / \epsilon_{1}-\gamma_{0}(p) / \epsilon_{0}}{\gamma_{1}(p) / \epsilon_{1}+\gamma_{0}(p) / \epsilon_{0}}
$$
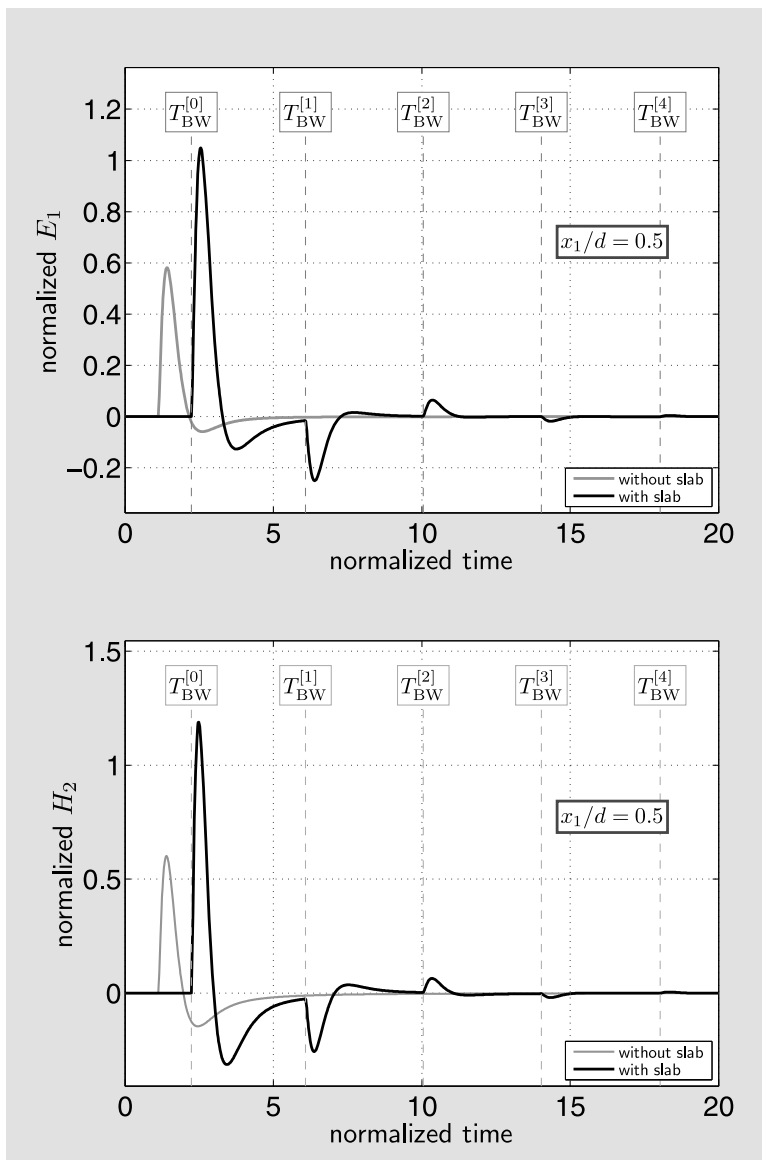

Figure 4. Time domain responses at $x_{1} / d=0.5$. The represented quantities are $E_{1} d / V_{\max }$ (normalized $E_{1}$ ), $\left(\mu_{0} / \epsilon_{0}\right)^{1 / 2} H_{2} d / V_{\max }$ (normalized $H_{2}$ ), and $c_{0} t / d$ (normalized time). 


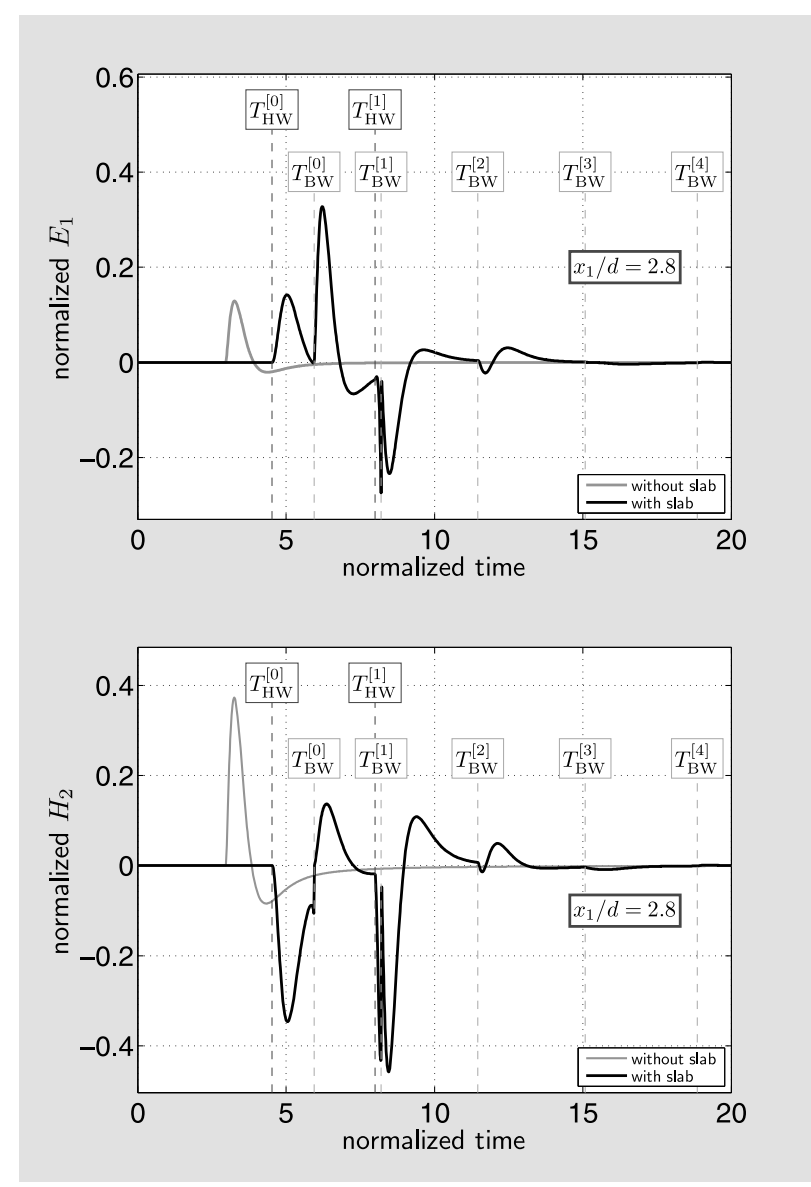

Figure 5. Time domain responses at $x_{1} / d=2.8$. The represented quantities are $E_{1} d / V_{\max }$ (normalized $E_{1}$ ), $\left(\mu_{0} / \epsilon_{0}\right)^{1 / 2} H_{2} d / V_{\max }$ (normalized $H_{2}$ ), and $c_{0} t / d$ (normalized time).

$$
\begin{gathered}
T_{H}(p)=\frac{2 \gamma_{1}(p) / \epsilon_{1}}{\gamma_{1}(p) / \epsilon_{1}+\gamma_{0}(p) / \epsilon_{0}} \\
\Delta=1-R_{H}(p) \exp \left[-2 s \gamma_{1}(p) d\right] .
\end{gathered}
$$

Via the convergent expansion

$$
\frac{1}{\Delta}=\sum_{n=0}^{\infty}\left[R_{H}(p)\right]^{n} \exp \left[-2 s n \gamma_{1}(p) d\right]
$$

the slowness-domain field quantities can be written as the superposition of constituents, each of which admits a analytic time domain representation attainable with Cagniard-De Hoop method.

[7] For convenience of the reader, the procedure as based on the time Laplace transformation as used by Cagniard [1939] and De Hoop [1960] is briefly reviewed in Appendix A. The corresponding analysis as based on the time Fourier transform, which can be found in the work of Chew [1990, section 4.2].

\section{The Time Domain Field at the Vacuum/ Dielectric Interface}

[8] In this section we focus on those field components at the interface $x_{3}=d$ that are continuous across this interface, i.e. $E_{1}$ and $H_{2}$. The radiated field in $\mathcal{D}_{0}$ is subsequently easily expressed in terms of these field values. Using the results of section 3 , we express them as

$$
\left\{\hat{E}_{1}, \hat{H}_{2}\right\}\left(x_{1}, d, s\right)=\sum_{n=0}^{\infty}\left\{\hat{E}_{1}^{[n]}, \hat{H}_{2}^{[n]}\right\}\left(x_{1}, d, s\right)
$$

with

$$
\begin{aligned}
\left\{\hat{E}_{1}^{[n]}, \hat{H}_{2}^{[n]}\right\}\left(x_{1}, d, s\right)= & \frac{s \hat{V}_{0}(s)}{2 \pi \mathrm{i}} \int_{p=-\mathrm{i} \infty}^{\mathrm{i} \infty}\left\{\gamma_{0}(p) / \epsilon_{0}, 1\right\} \\
& \cdot \frac{2}{\gamma_{1}(p) / \epsilon_{1}+\gamma_{0}(p) / \epsilon_{0}}\left[R_{H}(p)\right]^{n} \\
& \cdot \exp \left[-s p x_{1}-s(2 n+1) \gamma_{1} d\right] \mathrm{d} p
\end{aligned}
$$

The corresponding time domain expressions of each of these constituents follow upon the application of the Cagniard-DeHoop method (see Appendix A).

\section{Radiated Field in the Absence of a Dielectric Slab}

[9] To illustrate the pulse distortion that results from the presence of the dielectric slab we compare the relevant results with the ones applying to the radiation in the absence of the slab. The latter are well known:

$$
E_{1}\left(x_{1}, x_{3}, t\right)=\partial_{t} V_{0}(t) \stackrel{(t)}{*} \frac{1}{\pi} \frac{x_{3}}{r^{2}} \frac{t H\left(t-r / c_{0}\right)}{\left(t^{2}-r^{2} / c_{0}^{2}\right)^{1 / 2}}
$$




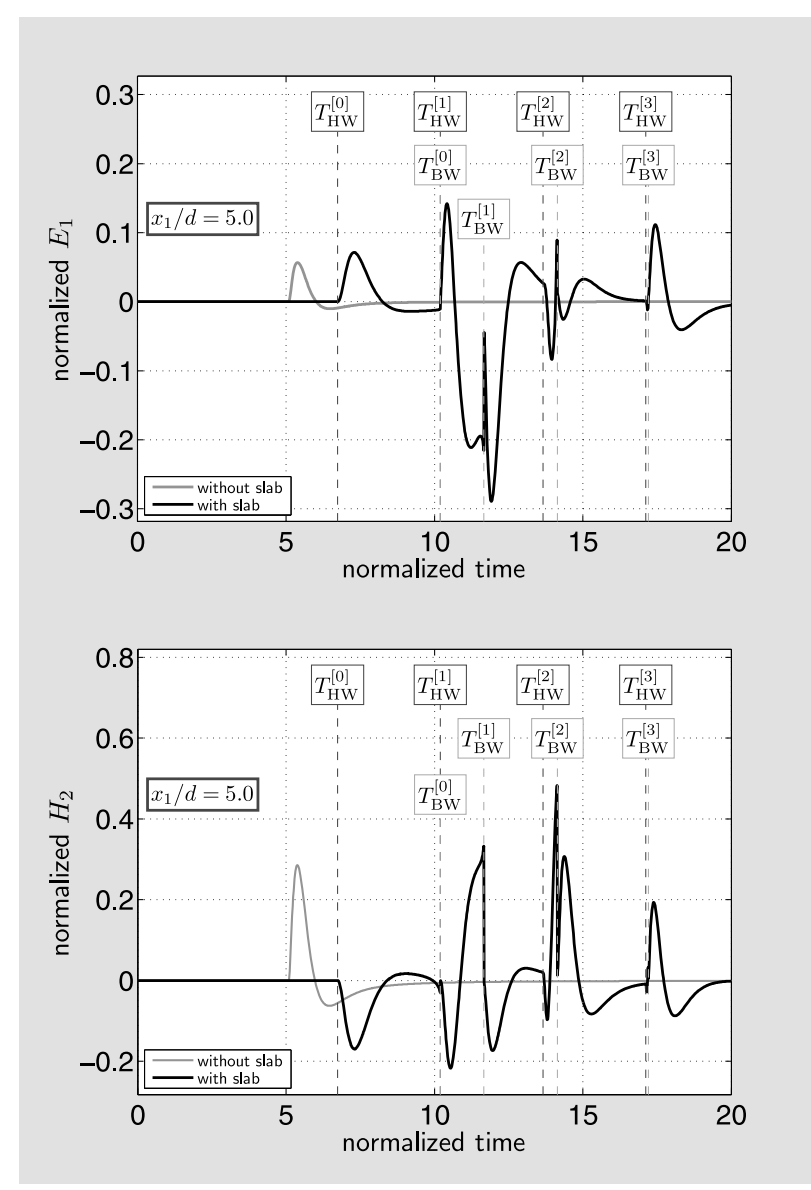

Figure 6. Time domain responses at $x_{1} / d=5.0$. The represented quantities are $E_{1} d / V_{\max }$ (normalized $\left.E_{1}\right),\left(\mu_{0} /\right.$ $\left.\epsilon_{0}\right)^{1 / 2} H_{2} d / V_{\max }$ (normalized $H_{2}$ ), and $c_{0} t / d$ (normalized time).

for $x_{3}>0$ and with $E_{1}\left(x_{1}, 0, t\right)=V_{0}(t) \delta\left(x_{1}\right)$, and

$$
\begin{gathered}
H_{2}\left(x_{1}, x_{3}, t\right)=\epsilon_{0} \partial_{t} V_{0}(t) \stackrel{(t)}{*} \frac{1}{\pi} \frac{H\left(t-r / c_{0}\right)}{\left(t^{2}-r^{2} / c_{0}^{2}\right)^{1 / 2}} \\
E_{3}\left(x_{1}, x_{3}, t\right)=-\partial_{t} V_{0}(t) \stackrel{(t)}{*} \frac{1}{\pi} \frac{x_{1}}{r^{2}} \frac{t H\left(t-r / c_{0}\right)}{\left(t^{2}-r^{2} / c_{0}^{2}\right)^{1 / 2}}
\end{gathered}
$$

where $^{*}$, denotes time convolution and $r=\left(x_{1}^{2}+x_{3}^{2}\right)^{1 / 2}>0$.

\section{Illustrative Numerical Results}

[10] This section contains some illustrative numerical results for the case of excitation with the power exponential pulse with parameters $c_{0} t_{w} / d=0.9236, t_{w} / t_{r}=$
$1.8473, \nu=2$, shown in Figure $2 \mathrm{a}$ (with Figure $2 \mathrm{~b}$ showing its spectral diagram) and vanishing slot width. In any finite time window of observation, only a finite number of terms in the summation in (28) yield a nonzero contribution, while in the range of critical refraction, only a subset of these contributions have a head wave part. The objective of our analysis is to compare the pulse shapes of the different constituents with the ones that the narrow slot antenna would radiate into a half-space with the properties of $\mathcal{D}_{0}$. This comparison is carried out on the vacuum/dielectric interface.

[11] The properties of the slab are taken as $\left\{\epsilon_{1}, \mu_{1}\right\}=$ $\left\{4 \epsilon_{0}, \mu_{0}\right\}$. All time convolution integrals contain inverse square-root singularities at one of the end points. These are numerically handled via a stretching of the variable of integration according to $\tau=T_{B W} \cosh (u)$, with $0<u<$ $\cosh ^{-1}\left(t / T_{B W}\right)$ for body wave constituents and $\tau=T_{B W} \sin$ $(v)$, with $\sin ^{-1}\left(T_{H W} / T_{B W}\right)<v<\pi / 2$ for head wave contributions. Four positions of observation at $x_{3}=d$ have been selected: (1) $x_{1} / d=0$, (2) $x_{1} / d=0.5$, (3) $x_{1} / d=2.8$, (4) $x_{1} / d=5.0$. In accordance with Figure 1, only the last two observation points are in the range of critical refraction. The time window of observation is taken as $0<$ $c_{0} t / d<20$. The arrival times of the different contributions are shown in Table 1.

[12] On account of the field equations, one might expect that in the first instance the pulse shapes of the field components would contain replicas and the time derivative of the exciting pulse shape. Outside the range of the occurrence of the head waves (in the frequency domain analysis of electromagnetic fields also denoted as lateral waves), this is more or less the case. In ranges where head wave contributions do occur, the situation is entirely different as shown in Figures 3-6. For pulse time widths of excitation that exceed the travel time to traverse the slab, the intermix of wave constituents overlapping in time leads to a signal in which the exciting pulse is no longer recognizable.

\section{Conclusion}

[13] Analytic time domain analytic expressions have been constructed for the head and body wave constituents of the time domain electromagnetic field radiated by a pulse-excited narrow slot in a perfectly conducting ground plane with a dielectric slab. Illustrative numerical results clearly show the changes in pulse shape arising from the multiple reflections, in particular in the range where head wave contributions occur. The results can be used to quantify these changes with a view of their acceptability in any transmission system where pulsed electromagnetic fields are the carrier of the information. In addition, the analytic time domain expressions can serve the purpose of providing benchmark results in the 
testing of purely computational software for the evaluation of time domain electromagnetic fields.

\section{Appendix A: The Cagniard-DeHoop Method}

[14] The generic form of the wave constituents in the interior of the dielectric slab and on its boundary is

$$
\begin{gathered}
\hat{w}\left(x_{1}, x_{3}, s\right)=\frac{s \hat{V}_{0}(s)}{2 \pi \mathrm{i}} \\
\int_{p=-\mathrm{i} \infty}^{\mathrm{i} \infty} \exp \left\{-s\left[p X+\gamma_{1}(p) Z\right]\right\} \tilde{w}(p) \mathrm{d} p,
\end{gathered}
$$

where $\tilde{w}(p)$ has the branch cuts in accordance with $\operatorname{Re}\left[\gamma_{0,1}(p)\right]>0$, i.e. $\left\{1 / c_{0,1}<|\operatorname{Re}(p)|<\infty, \operatorname{Im}(p)=0\right\}$, and $X$ and $Z$ are the propagation paths in the $x_{1}$ - and $x_{3}$-directions. We assume that $c_{0} \geqslant c_{1}$. Under the application of Cauchy's theorem and Jordan's lemma of complex function theory, the path of integration in (A1) is replaced with one along the modified Cagniard path (the Cagniard-DeHoop path) defined through

$$
p X+\gamma_{1}(p) Z=\tau \text { for } T<\tau<\infty,
$$

where $\tau$ is real-valued.

\section{A1. Body Wave Path}

[15] The body wave path follows from (A2) as the hyperbolic arc $\left\{p=p^{B W}(X, Z, \tau)\right\} \bigcup\left\{p=p^{B W^{*}}(X, Z\right.$, $\tau)\}$, where

$$
p^{B W}(X, Z, \tau)=\frac{X \tau+\mathrm{i} Z\left(\tau^{2}-T_{B W}^{2}\right)^{1 / 2}}{X^{2}+Z^{2}}
$$

for $T_{\mathrm{BW}}<\tau<\infty$ with

$$
T_{B W}=\left(X^{2}+Z^{2}\right)^{1 / 2} / c_{1}
$$

as the arrival time of the body wave constituent. Along this path

$$
\gamma_{1}\left(p^{B W}\right)=\frac{Z \tau-\mathrm{i} X\left(\tau^{2}-T_{B W}^{2}\right)^{1 / 2}}{X^{2}+Z^{2}}
$$

for $T_{\mathrm{BW}}<\tau<\infty$ and the Jacobian of the mapping from $p$ to $\tau$ is

$$
\frac{\partial p^{B W}}{\partial \tau}=\frac{\mathrm{i} \gamma_{1}\left(p^{B W}\right)}{\left(\tau^{2}-T_{B W}^{2}\right)^{1 / 2}}
$$

for $T_{\mathrm{BW}}<\tau<\infty$. The body wave path replaces the path of integration in (A1) as long as the intersection of this path with the real $p$ axes does not lie on the branch cut associated with $\gamma_{0}(p)$, i.e., for points of observation in the range $|X| /\left(X^{2}+Z^{2}\right)^{1 / 2}<c_{1} / c_{0}$. For points of observation outside this range, the body wave path has to be supplemented with a connecting loop integral along the branch cut associated with $\gamma_{0}(p)$. This loop integral yields the head wave (or lateral-wave) contribution to the wave constituent.

\section{A2. Head Wave Path}

[16] The parametrized head wave path follows from (A2) as the loop $\left\{p=p^{H W}(X, Z, \tau)\right\} \bigcup\left\{p=p^{H W^{*}}(X, Z, \tau)\right\}$, where

$$
p^{H W}(X, Z, \tau)=\frac{X \tau-Z\left(T_{B W}^{2}-\tau^{2}\right)^{1 / 2}}{X^{2}+Z^{2}}+\mathrm{i} 0
$$

for $T_{\mathrm{HW}}<\tau<T_{\mathrm{BW}}$ with

$$
T_{H W}=X / c_{0}+Z\left(1 / c_{1}^{2}-1 / c_{0}^{2}\right)^{1 / 2}
$$

[17] as the arrival time of the head wave constituent. Along this path

$$
\gamma_{1}\left(p^{H W}\right)=\frac{Z \tau+X\left(T_{B W}^{2}-\tau^{2}\right)^{1 / 2}}{X^{2}+Z^{2}}
$$

for $T_{\mathrm{HW}}<\tau<T_{\mathrm{BW}}$ and the Jacobian of the mapping from $p$ to $\tau$ is

$$
\frac{\partial p^{H W}}{\partial \tau}=\frac{\gamma_{1}\left(p^{H W}\right)}{\left(T_{B W}^{2}-\tau^{2}\right)^{1 / 2}}
$$

for $T_{\mathrm{HW}}<\tau<T_{\mathrm{BW}}$. The corresponding wave constituents follow from combining the two complex conjugate parts of the paths and applying Schwarz' theorem of complex function theory.

\section{A3. Time Domain Body Wave Constituent}

[18] The $s$-domain body wave constituent follows from (A1) as

$$
\begin{gathered}
\hat{w}(X, Z, s)=\frac{s \hat{V}_{0}(s)}{\pi} \int_{\tau=T_{B W}}^{\infty} \exp (-s \tau) \\
\operatorname{Im}\left[\tilde{w}\left(p_{B W}\right) \frac{\partial p^{B W}}{\partial \tau}\right] \mathrm{d} \tau .
\end{gathered}
$$


Again, on account of Lerch's uniqueness theorem of the unilateral Laplace transformation [Widder, 1946] the time domain constituent then follows as

$$
\begin{aligned}
w(X, Z, t)= & \partial_{t} V_{0}(t) \stackrel{(t)}{*} \frac{1}{\pi} \\
& \cdot\left\{\operatorname{Re}\left[\tilde{w}\left(p_{B W}\right) \gamma_{1}\left(p^{B W}\right)\right] \frac{H\left(t-T_{B W}\right)}{\left(t^{2}-T_{B W}^{2}\right)^{1 / 2}}\right\}
\end{aligned}
$$

where (A6) has been used and ${ }^{*}$, denotes convolution with respect to time.

\section{A4. Time Domain Head Wave Constituent}

[19] The $s$-domain head wave constituent follows from (A1) as

$\hat{w}(X, Z, s)=\frac{s \hat{V}_{0}(s)}{\pi} \int_{\tau=T_{H W}}^{T_{B W}} \exp (-s \tau) \operatorname{Im}\left[\tilde{w}\left(p^{H W}\right) \frac{\partial p^{H W}}{\partial \tau}\right] \mathrm{d} \tau$.

On account of Lerch's uniqueness theorem of the unilateral Laplace transformation [Widder, 1946] the time domain constituent then follows as

$$
\begin{gathered}
w(X, Z, t)=\partial_{t} V_{0}(t) \stackrel{(t)}{*} \frac{1}{\pi}\left\{\operatorname{Im}\left[\tilde{w}\left(p^{H W}\right) \gamma_{1}\left(p^{H W}\right)\right]\right. \\
\left.\cdot \frac{H\left(t-T_{H W}\right)-H\left(t-T_{B W}\right)}{\left(T_{B W}^{2}-t^{2}\right)^{1 / 2}}\right\}
\end{gathered}
$$

where (A10) has been used. These results are used in the main text.

\section{Appendix B: The Power Exponential Pulse}

[20] A convenient pulse type to model a unipolar pulse excitation is the power exponential pulse [Quak, 2001]:

$$
V_{0}(t)=V_{\max }\left(t / t_{\mathrm{r}}\right)^{\nu} \exp \left[-\nu\left(t / t_{\mathrm{r}}-1\right)\right] H(t)
$$

for $\nu=0,1,2, \ldots$ where $V_{\max }$ is the pulse amplitude, $\nu$ is the rising exponent of the pulse and $t_{\mathrm{r}}$ is the pulse risetime. Note that $V_{0}\left(t_{\mathrm{r}}\right)=V_{\text {max }}$. The pulse time width $t_{\mathrm{w}}$ follows from

$$
V_{\max } t_{\mathrm{w}}=\int_{t=0}^{\infty} V_{0}(t) \mathrm{d} t
$$

as

$$
t_{\mathrm{w}}=\left[(\nu-1) ! / \nu^{\nu}\right] t_{\mathrm{r}} \exp (\nu)
$$

The time Laplace transform of (B1) is

$$
\hat{V}_{0}(s)=\frac{V_{\max }}{t_{\mathrm{r}}^{\nu}} \frac{\nu !}{\left(s+\nu / t_{\mathrm{r}}\right)^{\nu+1}} \exp (\nu)
$$

The spectral amplitude of $V_{0}(t)$ follows from (B4) as

$$
\left|\hat{V}_{0}(\mathrm{i} \omega)\right|=\frac{V_{\max }}{t_{\mathrm{r}}^{\nu}} \frac{\nu !}{\left[\omega^{2}+\left(\nu / t_{\mathrm{r}}\right)^{2}\right]^{(\nu+1) / 2}} \exp (\nu) .
$$

From

$$
\left|\hat{V}_{0}(\mathrm{i} \omega) / \hat{V}_{0}(0)\right|=\frac{1}{\left[\left(\omega t_{\mathrm{r}} / \nu\right)^{2}+1\right]^{(\nu+1) / 2}}
$$

it follows that both

$$
\left|\hat{V}_{0}(\mathrm{i} \omega) / \hat{V}_{0}(0)\right| \leq 1
$$

and

$$
\left|\hat{V}_{0}(\mathrm{i} \omega) / \hat{V}_{0}(0)\right|<\frac{1}{\left(\omega t_{\mathrm{r}} / \nu\right)^{\nu+1}} .
$$

In the spectral diagram (where $\left|\hat{V}_{0}(\mathrm{i} \omega)\right|$ is plotted against $|\omega|$, both on logarithmic scales), the right-hand sides of (B7) and (B8) are straight lines that are denoted as the spectral bounds of $\left|\hat{V}_{0}(\mathrm{i} \omega) / \hat{V}_{0}(0)\right|$. The two spectral bounds intersect at their corner point

$$
\omega_{\text {corner }}=\nu / t_{\mathrm{r}}
$$

[21] Acknowledgments. The work described in this paper and the research leading to these results have received funding from the European Community's Seventh Framework Program FP7/20072013, under grant agreement 205294, HIRF SE project. The short term scientific mission of M. S. at International Research Centre for Telecommunications and Radar (IRCTR) has been financed by COST IC0603 ASSIST. The authors would like to thank the anonymous reviewers for their careful reading of the manuscript and for making valuable suggestions for improving the paper.

\section{References}

Cagniard, L. (1939), Réflexion et réfraction des ondes séismiques progressives, Gauthier-Villars, Paris.

Cagniard, L. (1962), Reflection and Refraction of Progressive Seismic Waves, translated from French by E. Flinn and C. H. Dix, McGraw-Hill, New York.

Chew, W. C. (1990), Waves and Fields in Inhomogeneous Media, Van Nostrand Reinhold, New York. 
De Hoop, A. T. (1960), A modification of Cagniard's method for solving seismic pulse problems, Appl. Sci. Res., Ser. B8, 349-356.

De Hoop, A. T. (1979), Pulsed electromagnetic radiation from a line source in a two-media configuration, Radio Sci., 14, 253-268.

De Hoop, A. T. (2000), Transient diffusive electromagnetic fields in stratified media: Calculation of the two-dimensional E-polarized field, Radio Sci., 35, 443-453.

De Hoop, A. T., and H. J. Frankena (1960), Radiation of pulses generated by a vertical electric dipole above a plane, nonconducting, earth, App. Sci. Res., Ser. B, 8, 369-377.

De Hoop, A. T., and M. L. Oristaglio (1988), Application of the modified Cagniard technique to transient electromagnetic diffusion problems, Geophys. J., 94, 387-397.

Langenberg, K. J. (1974), The transient response of a dielectric layer, Appl. Phys., 3, 179-188.
Quak, D. (2001), Analysis of transient radiation of a (traveling) current pulse on a straight wire segment, Proc. 2001 IEEE EMC Int. Sympo., 2, 849-854.

Widder, D. V. (1946), The Laplace Transform, pp. 63-65, Princeton Univ. Press, New Jersey.

A. T. de Hoop, Laboratory of Electromagnetic Research, Faculty of Electrical Engineering, Mathematics and Computer Science, Delft University of Technology, 4 Mekelweg, NL2628 CD Delft, Netherlands. (a.t.dehoop@tudelft.nl)

I. E. Lager, International Research Centre for Telecommunications and Radar, Faculty of Electrical Engineering, Mathematics and Computer Science, Delft University of Technology, 4 Mekelweg, NL-2628 CD Delft, Netherlands. (i.lager@tudelft.nl)

M. Štumpf, Department of Radio Electronics, Faculty of Electrical Engineering and Communication, Brno University of Technology, Purkynova 118, 61200 Brno, Czech Republic. (martin.stumpf@phd.feec.vutbr.cz) 